\title{
Adaptive Kalman Filter for MEMS-IMU based Attitude Estimation under External Acceleration and Parsimonious use of Gyroscopes
}

\author{
Aida Makni, Hassen Fourati, and Alain Kibangou
}

\begin{abstract}
This paper presents a viable quaternion-based Adaptive Kalman Filter (q-AKF) that is designed for rigid body attitude estimation. This approach is an alternative to overcome the limitations of the classical Kalman filter. The q-AKF processes data from a small inertial/magnetic sensor module containing triaxial gyroscopes, accelerometers, and magnetometers. The proposed approach addresses two challenges. The first one concerns attitude estimation during various dynamic conditions, in which external acceleration occurs. Although external acceleration is one of the main source of loss of performance in attitude estimation methods, this problem has not been sufficiently addressed in the literature. An adaptive algorithm compensating external acceleration from the residual in the accelerometer is proposed. At each step, the covariance matrix associated with the external acceleration is estimated to adaptively tune the filter gain. The second challenge is focused on the energy consumption issue of gyroscopes for long-term battery life of Inertial Measurement Units. We study the way to reduce the gyro measurement acquisition while maintaining acceptable attitude estimation. Through numerical simulations, under external acceleration and parsimonious gyroscope's use, the efficiency of the proposed $q-A K F$ is illustrated.
\end{abstract}

\section{INTRODUCTION}

Attitude determination, i.e. the determination of the three dimensional orientation of a rigid body, from non-ideal strap-down sensors, is a fundamental task and a crucial problem in a wide range of applications including ambulatory applications such as detection of unconstrained walking [1], pedestrian localization [2], [3], indoor navigation [4], and human body trackers [5], [6]. Extensive research on attitude estimation using compact MEMS-IMU (micro-electromechanical systems-inertial measurement units) has been performed since several decades. MEMS-IMU are principally composed of a triaxial gyroscope (to measure the angular velocity), a triaxial accelerometer (to measure the sum of the external acceleration and the gravity), and a triaxial magnetic sensor (to measure the Earth's magnetic field). Each of these sensors can be used separately to infer the attitude with relatively low precision. So, how to overcome the drawbacks that appear when each sensor is used separately and how to pertinently combine inertial and magnetic sensor measurements, are the key questions to be solved when devising an attitude estimation method.

According to the number of the involved sensors, attitude estimation methods in the literature can be cast into two

A. Makni, H. Fourati are with the Department of Automatic Control, Gipsa-Lab, UMR 5216, NeCS Team, Joseph Fourier University, Grenoble, France. (e-mails: aida.makni@gipsa-lab.fr, hassen.fouratiegipsa-lab.fr).

A. Kibangou is with the Department of Automatic Control, Gipsa-Lab, CNRS, NeCS Team, Joseph Fourier University, Grenoble, France. (e-mail: alain.kibangou@gipsa-lab.fr) groups. The first group makes use of two sensors, typically a magnetometer and an accelerometer, and includes deterministic methods. To find the attitude matrix, a classical least squares approach to the problem, as introduced by Wahba in [7], is in general considered [8], [9]. Those methods are devised with the reductive assumption that all movements are static or quasi-static; assumption that is not valid in all dynamic situations where external accelerations can be observed. In such cases, it becomes difficult to retrieve the attitude with high accuracy since it is not straightforward to dissociate external accelerations and the gravity. The second group of methods, which combines gyroscopes, accelerometers, and magnetometers, consists of classical filtering methods using either Kalman filters (KFs) [10], extended Kalman filters (EKFs) [11], or nonlinear observers [12]. The attitude estimation in these works is affected also by errors in dynamic situation, characterized by external acceleration.

Recently, some approaches consider the impact of external acceleration on the performance of attitude estimation [13]. An Adaptive Kalman Filter (AKF) can be adopted to fix such a problem. An adaptive EKF with an additive function noise in the measurement noise covariance matrix has been proposed in [13], [14]. However, it could not reflect the influence of accelerations on the observation covariance matrix accurately. In addition, those approaches require the setting of thresholds according to the system dynamics which is very tricky in practice. The authors of [15] addressed the combined estimation problem of the attitude and external acceleration by using an acceleration model-based approach. The proposed algorithm was capable of estimating accurate attitudes and external accelerations for short accelerated periods. Contrariwise, when the testing condition involved prolonged high external accelerations, the proposed algorithm exhibited gradually increasing errors. Recently, a novel quaternion Kalman filter (QKF) has been introduced by Choukroun et al. [16] where a detailed covariance analysis of the state-dependent noises has been developed. However, severe losses of performance are observed when using an accelerometer in its quaternion measurement model during dynamic motion cases. Therefore, how to consider more precisely the impact of external acceleration sensed by an accelerometer on the performance of the attitude estimation is still an open issue.

Using gyroscope measurements seems to be crucial to overcome limitation of accelerometers. However, gyroscopes are much more power consuming than accelerometers. As an example, the three-axis gyroscope L3GD20 of STMicro- 
electronics consumes $6.1 \mathrm{~mA}$ [17] while the consumption of the three-axis accelerometer LIS3DH is around $11 \mu \mathrm{A}$ [18]. For battery-operated applications using IMUs in attitude estimation, such as Pedestrian Navigation Systems, Smartphones, and monitoring systems for elderly or visually impaired persons, the energy consumption of gyroscopes is clearly a crucial issue. To the best to our knowledge, there is no work dealing with this issue in connection with attitude estimation performance. Nowadays, new generation of gyroscopes can be switched to operate in a low power mode where its outputs are turned-off, providing significant reduction of operating current (consumption around $1.5 \mathrm{~mA}$ ) [17]. Hence, it seems interesting to study how to decrease the use of gyro measurements by switching periodically or not to a low power mode without a significant loss of performance in the attitude estimation process.

To overcome the aforementioned difficulties, in this paper we propose:

- A quaternion-based adaptive Kalman Filter (q-AKF) compensating the external acceleration effect. The main advantage of the proposed method is that the observation covariance matrix is adaptively tuned according to the filter residual.

- A parsimonious use of gyroscope measurements during motion is added to the q-AKF by turning-off periodically the sensor and tuning accordingly the process covariance matrix.

This paper is organized as follows: In Section II, basic equations of the physical process and sensors are given. In Section III, the system's kinematic and observation models are presented, followed by the q-AKF under parsimonious use of gyroscopes. Simulation results are presented in Section IV. Eventually, Section V provides some conclusions and future works.

\section{BACKGROUND}

Let us consider a body-fixed frame $B\left(X_{B}, Y_{B}, Z_{B}\right)$ which has its origin at the triad of sensors and an Earth-fixed frame $N\left(X_{N}, Y_{N}, Z_{N}\right)$ (navigation frame or local level frame). $X_{B}$, $Y_{B}$, and $Z_{B}$ axes point along each of the triad of sensors. The $X$-axis points to the North. The $Z$-axis points towards the interior of the Earth, perpendicularly to the reference ellipsoid. The $Y$-axis completes the right-handed coordinate system, pointing East (NED: North, East, Down). The attitude of a rigid body can be represented using a quaternion $q=\left[\begin{array}{ll}q_{0} & \vec{q}^{T}\end{array}\right]^{T}=\left[\begin{array}{llll}q_{0} & q_{1} & q_{2} & q_{3}\end{array}\right]^{T} \in \mathbb{R}^{4}$, with $\|q\|=1$. According to [19], the dynamic of the system is described by the following equation:

$$
\dot{q}=\frac{1}{2} q \otimes \bar{\omega},
$$

where $\bar{\omega}=\left[\begin{array}{ll}0 & \omega^{T}\end{array}\right]^{T}$ is the quaternion representation of the angular velocity $\omega=\left[\begin{array}{lll}\omega_{x} & \omega_{y} & \omega_{z}\end{array}\right]^{T}$ expressed in $B$ and $\otimes$ denotes the quaternion product. For two unit-norm quaternions $q_{a}=\left[\begin{array}{ll}q_{a 0} & \vec{q}_{a}^{T}\end{array}\right]^{T}$ and $q_{b}=\left[\begin{array}{ll}q_{b 0} & \vec{q}_{b}^{T}\end{array}\right]^{T}$, the quaternion product is defined by:

$$
q_{a} \otimes q_{b}=\left[\begin{array}{cc}
q_{a 0} & -\vec{q}_{a}^{T} \\
\vec{q}_{a} & q_{a 0} I_{3 \times 3}+\left[\vec{q}_{a} \times\right]
\end{array}\right]\left[\begin{array}{c}
q_{b 0} \\
\vec{q}_{b}
\end{array}\right],
$$

where $I_{3 \times 3} \in \mathbb{R}^{3 \times 3}$ stands for the identity matrix while $\left[\vec{q}_{a}{ }^{\times}\right]$ represents the skew-symmetric matrix defined as:

$$
\left[\vec{q}_{a}^{\times}\right]=\left[\begin{array}{c}
q_{a, 1} \\
q_{a, 2} \\
q_{a, 3}
\end{array}\right]^{\times}=\left[\begin{array}{ccc}
0 & -q_{a, 3} & q_{a, 2} \\
q_{a, 3} & 0 & -q_{a, 1} \\
-q_{a, 2} & q_{a, 1} & 0
\end{array}\right]
$$

More details about quaternion can be found in [20].

The available MEMS sensors configuration in attitude estimation consists of a three-axis gyroscope, a three-axis accelerometer, and a three-axis magnetometer [21]. Their outputs $y_{g}, y_{a}$ and $y_{m} \in \mathbb{R}^{3}$ are respectively given by:

$$
\begin{aligned}
y_{g} & =\omega+\delta_{g}, \\
y_{a} & =C(q) G+a_{p}+\delta_{a}, \\
y_{m} & =C(q) m+\delta_{m},
\end{aligned}
$$

where $a_{p} \in \mathbb{R}^{3}$ denotes the external acceleration vector of the body, $G=\left[\begin{array}{lll}0 & 0 & g\end{array}\right]^{T}$ is the gravity vector $\left(g=9.81 \mathrm{~m} / \mathrm{s}^{2}\right)$, $m=\left[\begin{array}{lll}m_{x} & m_{y} & m_{z}\end{array}\right]^{T}=\left[\begin{array}{lll}\|m\| \cos \theta & 0 & \|m\| \sin \theta\end{array}\right]^{T}$ represents the Earth's magnetic field vector measured in $N$. The theoretical model of magnetic field vector nearest to reality is given in [22] and considers a magnetic field with an inclinaison angle $\theta=60^{\circ}$ and a vector norm $\|m\|=0.5$ Gauss. $y_{g}$ can be often corrupted with a small bias. $C(q)$ is the rotation matrix, rotating a vector $\vec{n}$ in $N$ to $B$, defined as:

$$
C(q)=\left[\begin{array}{ccc}
2 q_{0}^{2}+2 q_{1}^{2}-1 & 2 q_{1} q_{2}+2 q_{0} q_{3} & 2 q_{1} q_{3}-2 q_{0} q_{2} \\
2 q_{1} q_{2}-2 q_{0} q_{3} & 2 q_{0}^{2}+2 q_{2}^{2}-1 & 2 q_{2} q_{3}+2 q_{0} q_{1} \\
2 q_{1} q_{3}+2 q_{0} q_{2} & 2 q_{2} q_{3}-2 q_{0} q_{1} & 2 q_{0}^{2}+2 q_{3}^{2}-1
\end{array}\right]_{(7)}
$$

Sensor noises $\delta_{g}, \delta_{a}$, and $\delta_{m}$ are assumed to be zeromean white Gaussian noises mutually uncorrelated with the following covariance matrices $R_{g} I_{3 \times 3}, R_{a} I_{3 \times 3}$, and $R_{m} I_{3 \times 3}$, respectively.

\section{QuATERnion AdAPtive KaLMAn Filter With PARSIMONIOUS GYRO DATA}

\section{A. The process model}

Equation (1) describes the time rate of attitude variation as a result of rigid body angular rates measured by the gyroscope. The discrete-time process of the attitude kinematics which corresponds to (1) is given by [16]:

$$
q_{k+1}=\Phi_{k} q_{k}+w_{k}
$$

where $q_{k}$ is the quaternion representing the attitude, $\Phi_{k}$ is the matrix containing the angular velocity vector $y_{g, k}$ :

$$
\begin{gathered}
\Phi_{k}=\exp \left(\Omega_{k} \Delta t\right), \\
\Omega_{k}=\frac{1}{2}\left[\begin{array}{cc}
0 & -y_{g_{g, k}}^{T} \\
y_{g, k} & -\left[y_{g, k}^{\times}\right]
\end{array}\right],
\end{gathered}
$$


$\Delta t$ is the sampling period and $w_{k} \sim \mathscr{N}\left(0, Q_{k}\right)$ is the process noise defined as:

$$
\begin{gathered}
w_{k}=-\frac{\Delta t}{2} \Xi_{k} \delta_{g, k}, \\
\Xi_{k}=\left[\begin{array}{c}
-\vec{q}_{k}^{T} \\
{\left[\vec{q}_{k}{ }^{\top}\right]+q_{0, k} I_{3 \times 3}}
\end{array}\right] .
\end{gathered}
$$

As mentioned in Section I, the filter is elaborated with parsimonious use of gyro measurements. The approach consists in shutting-down the gyroscope over a fixed number of samples and keeping the last angular velocity value in the process model, periodically. Then, for more convenience, the process model (8) is modified as follows:

$$
q_{k+1}=\gamma_{k} \Phi_{k} q_{k}+\left(1-\gamma_{k}\right) \Phi_{k}^{\text {last }} q_{k}+\gamma_{k} w_{k}+\left(1-\gamma_{k}\right) \bar{w}_{k},
$$

where $\gamma_{k}$ can take two values: If gyroscope measurements are available then $\gamma_{k}=1$ else $\gamma_{k}=0$, and $\Phi_{k}^{\text {last }}$ contains the last value of angular velocity $y_{g, k}^{\text {last }}$ taken at the sample before shutting-down the gyroscope:

$$
\Phi_{k}^{\text {last }}=\exp \left(\Omega_{k}^{\text {last }} \Delta t\right),
$$

and

$$
\Omega_{k}^{\text {last }}=\frac{1}{2}\left[\begin{array}{cc}
0 & -\left(y_{g, k}^{\text {last }}\right)^{T} \\
y_{g, k}^{\text {last }} & -\left[y_{g, k}^{\text {last }}\right]
\end{array}\right] .
$$

$\bar{w}_{k} \sim \mathscr{N}\left(0, \hat{Q}_{k}\right)$ is the process noise vector when angular velocity measurements are absent. In this case, we impose an exponential variation to the covariance matrix $\hat{Q}_{k}$ during missing data periods:

$$
\hat{Q}_{k}=Q_{k}^{\text {last }} \exp (\alpha k \Delta t)
$$

with $\alpha$ is a constant that should be suitably ajusted and $Q_{k}^{\text {last }}$ is the covariance matrix corresponding to the last angular velocity value taken at the sample before shutting-down the gyroscope.

\section{B. Observation model}

Let us consider the quaternion vectors $Y_{a}=\left[\begin{array}{ll}0 & y_{a}^{T}\end{array}\right]^{T}$, $\bar{G}=\left[\begin{array}{ll}0 & G^{T}\end{array}\right]^{T}, Y_{m}=\left[\begin{array}{ll}0 & y_{m}^{T}\end{array}\right]^{T}$ and $\bar{m}=\left[\begin{array}{ll}0 & m^{T}\end{array}\right]^{T}$ associated respectively with $y_{a}, G, y_{m}$, and $m$.

It is well known that $Y_{a}$ and $Y_{m}$, in discrete time, are related by the quaternion of rotation $q_{k}$ as follows:

$$
\begin{aligned}
Y_{a, k} & =q_{k}^{-1} \otimes \bar{G} \otimes q_{k} \\
Y_{m, k} & =q_{k}^{-1} \otimes \bar{m} \otimes q_{k}
\end{aligned}
$$

where $q_{k}^{-1}=\left[\begin{array}{llll}q_{0, k} & -q_{1, k} & -q_{2, k} & -q_{3, k}\end{array}\right]^{T}$ is the complementary quaternion. Left multiply $q_{k}$ on both sides of (17) and (18) and do the difference between both sides in each obtained equation, lead to the following quaternion pseudoobservation model [16]:

$$
0_{4 \times 1}=H_{k} q_{k}+v_{k},
$$

where

$$
H_{k}=\left(\begin{array}{c}
H_{1, k} \\
H_{2, k}
\end{array}\right)
$$

with

$$
\begin{gathered}
H_{1, k}=\left(\begin{array}{cc}
0 & -\left(y_{a, k}-G\right)^{T} \\
\left(y_{a, k}-G\right) & -\left[\left(y_{a, k}+G\right)^{\times}\right]
\end{array}\right), \\
H_{2, k}=\left(\begin{array}{cc}
0 & -\left(y_{m, k}-m\right)^{T} \\
\left(y_{m, k}-m\right) & -\left[\left(y_{m, k}+m\right)^{\times}\right]
\end{array}\right),
\end{gathered}
$$

and

$$
v_{k}=\left(\begin{array}{c}
w_{a c c, k}^{q} \\
w_{m g, k}^{q}
\end{array}\right)=-\frac{1}{2} \Xi\left(q_{k}\right)\left(\begin{array}{c}
a_{p, k}+\delta_{a, k} \\
\delta_{m, k}
\end{array}\right) .
$$

One can note that quaternion-dependent noise, related to magnetometer $w_{m g, k}^{q} \sim \mathscr{N}\left(0, R_{m g, k}\right)$, is known. However, $w_{a c c, k}^{q} \sim \mathscr{N}\left(r_{k}, R_{a c c, k}\right)$, related to accelerometer is unknown when external acceleration are detected. Therefore, $r_{k}$ and $R_{a c c, k}$ will be adaptively estimated in the filter.

\section{Filter design}

Once the process and observation models are defined as the above, the procedure of the proposed q-AKF can be summarized as follows:

1) Initialize the state estimate $\hat{q}_{0 / 0}$ and the error covariance matrix $P_{0 / 0}$.

2) Compute the a priori state estimate

$$
\hat{q}_{k / k-1}=\left[\gamma_{k} \Phi_{k}+\left(1-\gamma_{k}\right) \Phi_{k}^{\text {last }}\right] \hat{q}_{k-1 / k-1} .
$$

3) Compute the a priori error covariance estimate

$$
\begin{aligned}
P_{k / k-1}= & {\left[\gamma_{k} \Phi_{k}+\left(1-\gamma_{k}\right) \Phi_{k}^{\text {last }}\right] P_{k-1 / k-1} } \\
& {\left[\gamma_{k} \Phi_{k}+\left(1-\gamma_{k}\right) \Phi_{k}^{\text {last }}\right]^{T}+\left[\gamma_{k} Q_{k}+\left(1-\gamma_{k}\right) \hat{Q_{k}}\right], }
\end{aligned}
$$

where

$$
Q_{k}=\frac{\Delta t^{2}}{4} \Xi\left(q_{k}\right) R_{g} \Xi\left(q_{k}\right)^{T},
$$

and $\hat{Q}_{k}$ is given in (16).

4) Compute the Kalman gain

$$
K_{k}=P_{k / k-1} H_{k}\left(H_{k} P_{k / k-1} H_{k}^{T}+R_{k}\right)^{-1},
$$

where

$$
R_{k}=\left(\begin{array}{c}
R_{m g, k} \\
\hat{R}_{a c c, k}
\end{array}\right)
$$

and

$$
R_{m g, k}=\frac{1}{4} \Xi\left(\hat{q}_{k / k-1}\right) R_{m} \Xi\left(\hat{q}_{k / k-1}\right)^{T} .
$$

5) Compute the a posteriori state estimate

$$
\hat{q}_{k / k}=\left(I-K_{k} H_{k}\right) \hat{q}_{k / k-1} .
$$

6) Compute the a posteriori error covariance estimate

$$
P_{k / k}=\left(I-K_{k} H_{k}\right) P_{k / k-1} .
$$

The estimated covariance $\hat{R}_{a c c, k}$ in (28) is computed using an adaptive approach [23] to compensate the external acceleration. 
TABLE I

CHARACTERISTICS OF THE VARIOUS NOISES FOR SENSOR MEASUREMENTS

\begin{tabular}{llll}
\hline \hline Sensors & Parameters & $\begin{array}{l}\text { Standard } \\
\text { deviations }\end{array}$ & Units \\
\hline Accelerometer & $\delta_{a}$ & 0.05 & $\mathrm{~m} / \mathrm{s}^{2}$ \\
Magnetometer & $\delta_{m}$ & 0.05 & $\mathrm{Gauss}$ \\
Gyroscope & $\delta_{g}$ & 0.05 & $\mathrm{rad} / \mathrm{s}$ \\
\hline
\end{tabular}

Online estimation of $\hat{R}_{a c c, k}$ : The external acceleration $a_{p, k}$ is unknown in the observation noise (23). Then, we suggest to estimate its covariance matrix $\hat{R}_{a c c, k}$ from the residual in the accelerometer measurement update $r_{k}=-H_{1, k} \hat{q}_{k / k-1}$. Given $N$ consecutive observations from $k=k_{0}-N+1$ to $k=k_{0}$, an unbiased estimate of the mean is given by:

$$
\hat{r}_{k}=\frac{1}{N} \sum_{j=k-N+1}^{k} r_{j}
$$

Following the method in [23], the unbiased estimation of $\hat{R}_{a c c, k}$ can be obtained such as:

$$
\hat{R}_{a c c, k}=\frac{1}{2} \sum_{j=k-N+1}^{k}\left(r_{j}-\hat{r}_{k}\right)\left(r_{j}-\hat{r}_{k}\right)^{T}-\frac{N-1}{N} H_{1, j} P_{j / j-1} H_{1, j}^{T} .
$$

\section{Simulation Results}

This section aims to illustrate the performance of the designed q-AKF. Some numerical simulations were carried out to estimate a rigid body attitude based on theoretical inertial and magnetic measurements. We considered an attitude variation example taken from angular velocity data over $30 \mathrm{~s}$. The following angular rate values issued from the gyroscope are simulated:

$$
\left\{\begin{array}{l}
\omega_{x}(t)=2 \cos (1.5 t) \\
\omega_{y}(t)=-2 \sin (0.9 t) \\
\omega_{z}(t)=1.5 \cos (1.2 t)
\end{array}\right.
$$

Then, the kinematic equation (1) and angular velocity $\omega$ given by (34) were used to generate a quaternion $q$ used as a reference to compare it with the estimated quaternion from the q-AKF. Moreover, the theoretical outputs of accelerometer and magnetometer are created using (5), (6), respectively, and the rotation matrix in (7) (computed using the theoretical quaternion). To represent the sensor imperfections, an additive random zero-mean white Gaussian noise was considered for all sensor measurements, with the same standard deviation (see Table I). A high external acceleration is added to the accelerometer output. The sampling rate was chosen as $100 \mathrm{~Hz}$ for all measurements.

The theoretical components of the quaternion (reference) as well as those of the q-AKF were initialized with different random values which were summarized in Table II. Notice that this choice allows us to illustrate the convergence of the filter even though it was initialized far from the actual states. The initial estimation error covariance matrix is chosen such as:
TABLE II

INITIAL CONDITIONS

\begin{tabular}{cccc}
\hline \hline Theoretical model & $q(0)=\left[\begin{array}{llll}0.3 & -0.6 & 0.75 & 0.1\end{array}\right]^{T}$ \\
q-AKF & $\hat{q}(0)=\left[\begin{array}{llll}1 & 0 & 0 & 0\end{array}\right]^{T}$ \\
\hline
\end{tabular}

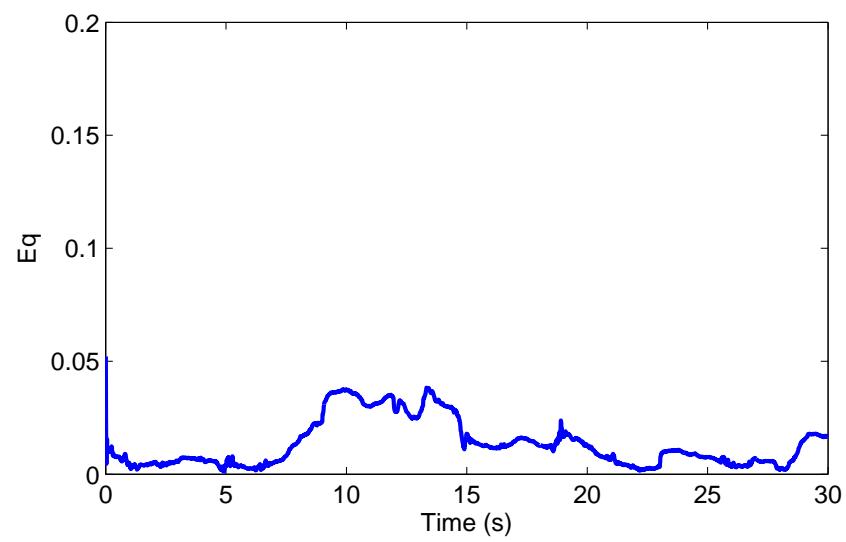

Fig. 1. Quaternion estimation error norm

$$
P_{0 / 0}=0.1 I_{4 \times 4}
$$

Monte-Carlo simulations of 100 independent runs was realized in all simulation cases. For online estimation of $\hat{R}_{a c c, k}$ as described in (33), $N$ was set equal to 100 .

\section{A. Adaptive filter performances with $\gamma=1$}

We consider the case where we have full gyro measurements i.e. $\gamma=1$. In order to evaluate the overall performance of the attitude estimation, we plotted the evolution of the quaternion estimation error using the following equation:

$$
E_{q, k}=\left\|\left(q_{k}^{-1} \otimes \hat{q}_{k}\right)-1\right\|
$$

Fig. 1 depicts the convergence of this error towards zero during the simulated motion. Fig. 2 shows the time history evolution of the Euler angles (roll, pitch and yaw) estimated by q-AKF and the QKF proposed by Choukroun et al. [16]. We chose to express the attitude in Euler angles representation because it is more intuitive than quaternion for the reader. The mathematical transformation between the quaternion and Euler angles was given in [24]. The results show that the q-AKF can provide the best performance even during the high external acceleration phases, for example during the interval (23-30 s). Moreover, the filter performance was shown quantitatively using the Root Mean Square Error (RMSE) which can be written such as:

$$
R M S E=\sqrt{\frac{1}{T} \sum x^{2}(t)}
$$

where $T$ is the time interval and $x$ is the computed error. The RMSE from the q-AKF and the QKF are given in Table III. 

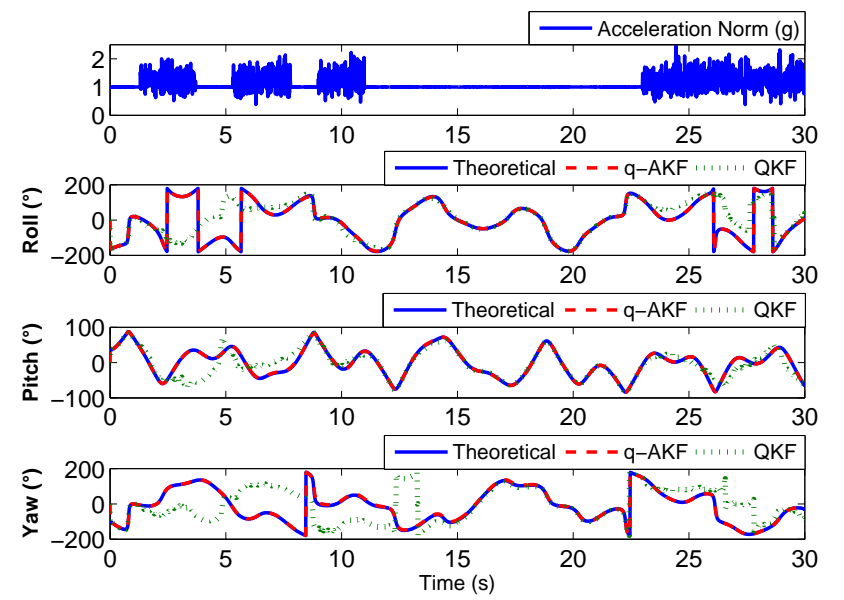

Fig. 2. Euler Angle Estimation

TABLE III

RMSE OF EULER ANGLES ESTIMATION (Q-AKF VS QKF)

\begin{tabular}{cccc}
\hline \hline & Roll $\left(^{\circ}\right)$ & Pitch $\left(^{\circ}\right)$ & Yaw $\left(^{\circ}\right)$ \\
\hline q-AKF & 12.6233 & 3.0214 & 8.9228 \\
\hline QKF & 38.5725 & 10.3664 & 24.4625 \\
\hline
\end{tabular}

\section{B. Adaptive filter performances with $\gamma=0$}

In this case, we considered the scenario where the gyroscope is in active mode for a period of $M_{1}=20$ samples followed by a period of $M_{2}=20$ samples where the sensor is turned-off (low power mode) (see Fig. 3). This scenario is repeated periodically during the simulation test. At the end of $M_{1}$, we use the last angular velocity value in the next steps during $M_{2}$ where the error process covariance matrix $\hat{Q}_{k}$ was computed as in (16) with $\alpha=20$. The results in Euler angles are given in Fig. 4 and Table IV in static motion. It can be seen that the q-AKF still robust even 50\% of gyroscope observations are lost (1500 from 3000 samples).

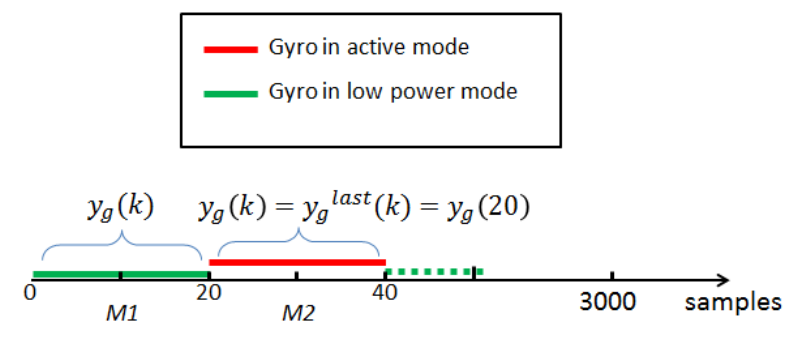

Fig. 3. Gyro acquisition scenario

To give some insight about reducing gyroscope's use, we tested the q-AKF with different parsimonious use of gyroscope measurements by varying the period size of $M_{2}$. We defined a scalar $\sigma$ describing the variation of RMSE values under the number of gyro acquisitions $l$ during simulation
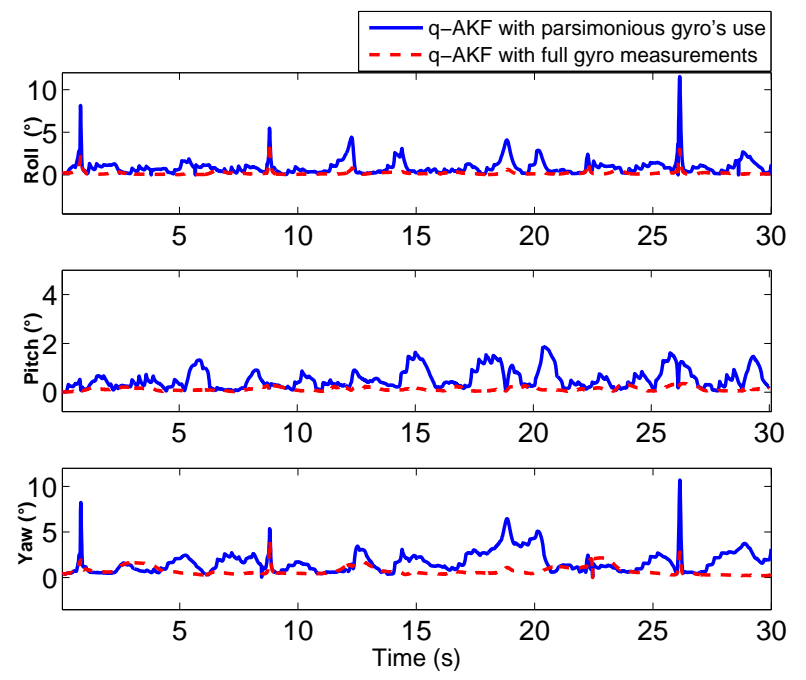

Fig. 4. Euler Angles Estimation Errors

TABLE IV

ROOT MEAN SQUARE ERRORS OF EULER ANGLES ESTIMATION IN STATIC MOTION

\begin{tabular}{cccc}
\hline \hline & Roll $\left({ }^{\circ}\right)$ & Pitch $\left({ }^{\circ}\right)$ & Yaw $\left(^{\circ}\right)$ \\
\hline q-AKF with parsimonious gyro's use & 15.7020 & 3.2984 & 12.8401 \\
\hline q-AKF with full gyro measurements & 11.3820 & 2.3701 & 7.3051 \\
\hline
\end{tabular}

test, such as:

$$
\sigma=\frac{R M S E}{l} .
$$

Fig. 5 shows the $\sigma$ evolution according to various sizes of $M_{2}$. This figure represents a compromise between the estimation accuracy of the q-AKF and the size of low power mode period $M_{2}$. We conclude that estimation performance decreases starting from $M_{2}=30$ samples. However, this period is still an interesting improvement about the use of gyroscope.

\section{CONCLUSION}

In this paper, we have presented the design of a q-AKF for rigid body attitude estimation using inertial/magnetic sensors, i.e. accelerometers, gyroscopes, and magnetometers. The q-AKF was designed with the goal of being able to produce accurate attitude estimates under external acceleration and parsimonious use of gyroscope measurements. The filter was developed with two major contributions. The first one is to improve the compensation of external acceleration. The proposed filter does not need the setting of thresholds or the modelling of external acceleration. Based on the filter residual in the accelerometer, the external acceleration covariance matrix is estimated to adaptively tune the filter gain. The second contribution is related to the energy consumption issue of gyroscopes for long-term battery life of IMUs. The q-AKF was modified to find the best way to parsimoniously use the gyroscope measurement by turning-off and activating 


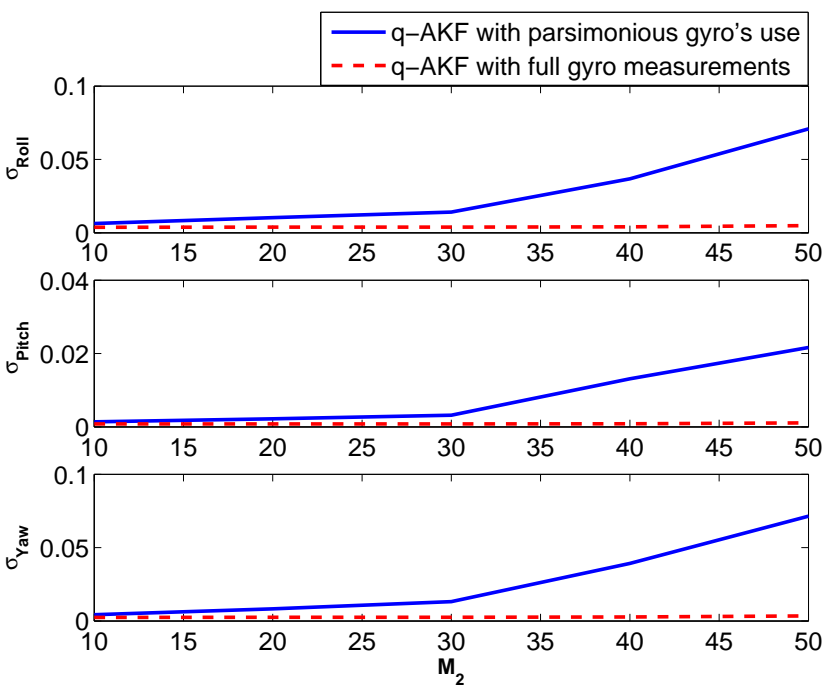

Fig. 5. Comparaison of $\sigma$ of Euler angles (q-AKF with parsimonious gyro's use in solid line Vs q-AKF with full gyro measurements in dashed line)

the output of the sensor alternatively, while maintaining acceptable attitude estimation. The process noise covariance is adaptively tuned for optimal compensation of the error. Future works will focus on a better way for intermittently use of gyroscope to improve the attitude estimation and on experimental evaluation of the proposed schemes.

\section{REFERENCES}

[1] J.-K. Lee and E. J. Park, "Quasi real-time gait event detection using shank-attached gyroscopes." Med. Biol. Engineering and Computing, vol. 49, no. 6, pp. 707-712, 2011.

[2] D. Roetenberg, P. J. Slycke, and P. H. Veltink, "Ambulatory position and orientation tracking fusing magnetic and inertial sensing," IEEE Transactions on Biomedical Engineering, vol. 54, no. 5, pp. 883-890, 2007.

[3] X. Yun, E. Bachmann, H. Moore, and J. Calusdian, "Self-contained position tracking of human movement using small inertial/magnetic sensor modules," in IEEE International Conference on Robotics and Automation, april 2007, pp. 2526-2533.

[4] E. Bachmann, I. Duman, U. Y. Usta, R. Mcghee, X. Yun, and M. Zyda, "Orientation tracking for humans and robots using inertial sensors," in Computational Intelligence in Robotics and Automation, 1999, pp. 187-194.

[5] E. Foxlin, "Inertial head-tracker sensor fusion by a complimentary separate-bias kalman filter," in Proceedings of the Virtual Reality Annual International Symposium. Washington, DC, USA: IEEE Computer Society, 1996, pp. 185-194.

[6] W. M. S., W. Conrad, R. Jimmy, O. Edward, S. Kathleen, and F. Robert, "Tilt determination in mems inertial vestibular prosthesis," Journal of biomechanical engineering, vol. 128, no. 6, pp. 943-956, 2006.

[7] G. Wahba, "A least squares estimate of satellite attitude," SIAM Review, vol. 7, no. 3, Jul. 1965.

[8] D. Gebre-Egziabher, G. Elkaim, J. D. Powell, and B. Parkinson, "A gyro-free quaternion-based attitude determination system suitable for implementation using low cost sensors," in Position Location and Navigation Symposium, IEEE 2000, 2000, pp. 185-192.

[9] M. S. E.J Lefferts, F.L Markley, "Kalman filtering for spacecraft attitude estimation," Journal of Guidance, Control and Dynamics, vol. 5, no. 5, pp. 417-429, September - October 1982.

[10] R. Zhu, D. Sun, Z. Zhou, and D. Wang, "A linear fusion algorithm for attitude determination using low cost mems-based sensors," Measurement, vol. 40, no. 3, pp. 322-328, 2007.
[11] S. Bonnabel, "Left-invariant extended kalman filter and attitude estimation," in 46th IEEE Conference on Decision and Control, 2007, pp. $1027-1032$.

[12] P. Martin and E. Salaun, "Invariant observers for attitude and heading estimation from low-cost inertial and magnetic sensors," in IEEE Conference on Decision and Control, 2007, pp. 1039-1045.

[13] J. W. Wei Li, "Effective adaptive kalman filter for mems$\mathrm{imu}$ /magnetometers integrated attitude and heading reference systems," Journal of Navigation, vol. 66, no. 01, pp. 99-113, January 2013.

[14] R. Munguia and A. Grau, "Attitude and heading system based on ekf total state configuration," in IEEE International Symposium on Industrial Electronics (ISIE), 2011, pp. 2147-2152.

[15] J. K. Lee, E. Park, and S. Robinovitch, "Estimation of attitude and external acceleration using inertial sensor measurement during various dynamic conditions," IEEE Transactions on Instrumentation and Measurement, vol. 61, no. 8, pp. 2262-2273, 2012.

[16] D. Choukroun, I. Y. Bar-Itzhack, and Y. Oshman, "Novel quaternion kalman filter," IEEE Transactions on Aerospace and Electronic Systems, vol. 42, no. 1, pp. 174-190, 2006.

[17] STMicroelectronics, "Mems motion sensor:three-axis digital output gyroscope," STMicroelectronics, Tech. Rep., 2013.

[18] STMicroelectronics., "Mems digital output motion sensor ultra lowpower high performance three-axis nano accelerometer," STMicroelectronics, Tech. Rep., 2013.

[19] M. D. Shuster, "Survey of attitude representations," Journal of the Astronautical Sciences, pp. 439-517, Oct.

[20] J. B. Kuipers, Quaternions and rotation sequences : a primer with applications to orbits, aerospace, and virtual reality. Princeton, $\mathrm{NJ}$ Princeton Univ. Press, 1999.

[21] S. Beeby, MEMS Mechanical Sensors. Artech House, 2004.

[22] Astrosurf. [Online]. Available: http://www.astrosurf.com

[23] K. Myers and B. Tapley, "Adaptive sequential estimation with unknown noise statistics," IEEE Transactions on Automatic Control, vol. 21, no. 4, pp. 520-523, 1976.

[24] C. E. H. W. F. Phillips and G. A. Gebert, "Review of attitude representations used for aircraft kinematics," J. Aircraft, vol. 38, no. 04 , pp. $718-223,2001$ 